\title{
A trade-off study of lunar-earth optical communication links
}

\section{Tomohiro Araki}

Tomohiro Araki, "A trade-off study of lunar-earth optical communication links," Proc. SPIE 11852, International Conference on Space Optics - ICSO 2020, 118521X (11 June 2021); doi: 10.1117/12.2599331

SPIE Event: International Conference on Space Optics - ICSO 2021, 2021, Online SPIE. Only 


\section{International Conference on Space Optics-ICSO 2020}

Virtual Conference

30 March-2 April 2021

Edited by Bruno Cugny, Zoran Sodnik, and Nikos Karafolas
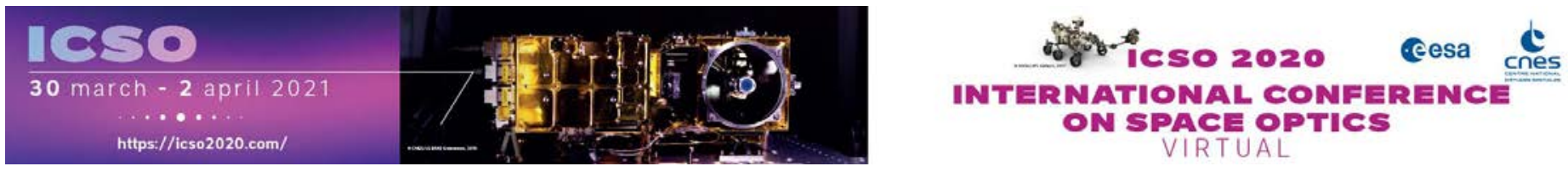

A trade-off study of lunar-earth optical communication links

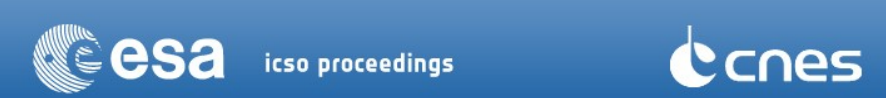

International Conference on Space Optics - ICSO 2020, edited by Bruno Cugny, Zoran Sodnik, Nikos Karafolas, Proc. of SPIE Vol. 11852, 118521X · @ 2021 ESA and CNES

CCC code: $0277-786 \mathrm{X} / 21 / \$ 21 \cdot$ doi: $10.1117 / 12.2599331$ 


\title{
A Trade-Off Study of Lunar-Earth Optical Communication Links
}

\author{
Tomohiro Araki* \\ Japan Aerospace Exploration Agency (JAXA) \\ 2-1-1 Sengen, Tsukuba-city, Ibaraki, JAPAN 305-8505
}

\begin{abstract}
A new era of manned lunar exploration is dawning. Optical communication technology is expected to become indispensable for high-speed data transmission from lunar explorers to the Earth. This paper describes the expectations for contents from the Moon and the estimated amount of information. Then, two types of Lunar-Earth optical communication systems are discussed, including a comparison between direct communication with an optical ground station (OGS) and communication through a data relay satellite in geostationary orbit (GEO) around the Earth.
\end{abstract}

Keywords: Optical Communications, Moon mission, PSK

\section{INTRODUCTION}

The USA (NASA), EU (ESA), Russia (Roscosmos), Canada (CSA), Japan (JAXA), and other agencies have agreed to cooperate in manned exploration of the Moon and in developing a Lunar Orbiting Platform-Gateway (hereafter Gateway) in the late $2020 \mathrm{~s}^{1}$. In a large-capacity data transmission from Gateway and lunar explorers to the Earth, a transmission speed of several hundred megabits per second (Mbps) to some gigabits per second (Gbps) will be needed. However, transmission speed of some Gbps is difficult to realize by using radio frequency (RF) technology, thus optical communications are expected to be indispensable tools for the future lunar exploration.

Like communications with NASA's LADEE lunar explorer ${ }^{2}$, an optical communication system between the Earth and the Moon would use uplink beacons from an optical ground station (OGS) to a spacecraft on and around the Moon and downlink optical signal back. However, such system has a difficulty that optical signals are blocked by clouds. Moreover, the coverage is also problematic. Considering the Earth's rotation, at least three OGSs for worldwide coverage for communicating between an OGS and a lunar spacecraft through Gateway, just like as NASA's famous Deep Space Network (DSN) ${ }^{3}$. This would enable a high operation rate only in the ideal condition with one antenna and an RF system not affected by the atmosphere, such as the $\mathrm{S}$ and $\mathrm{X}$ bands. However, in practice, ten or more OGSs located in remote locations worldwide would be needed to establish a reliable operational optical link between the Moon and the Earth.

On the other hand, an optical communication terminal in space is not affected by the atmosphere. An optical communication terminal on a data relay satellite in geosynchronous orbit (GSO) around the Earth can reliably perform optical communication for almost half of each day.

This paper is organized as follows. Section 2 discusses the expected type and amount of mission data sent between the Moon and the Earth. This is a statement of the fundamental requirements for an optical up- or downlink (between the Moon to the Earth). The author reports on a preliminary study of optical return links, including an estimated link budget in section 3. Both links between the Moon and the Earth, with and without a data relay satellite in GEO, are discussed. Section 4 is a study of the trade-off between using several OSGs or several relay satellites. Section 5 is the summary and conclusion of this paper.

*tomohiro.araki@jaxa.jp 


\section{EXPECTATIONS: THE TYPE AND VOLUME OF LUNAR MISSION DATA}

Except for tangible samples such as rocks, sand, and dust, all extraterrestrial results are in the form of acquired data, which is transmitted from the spacecraft. Also, commands are communicated to the spacecraft. The communication and power subsystems are essential components of the spacecraft infrastructure. This section considers the requirements for optical data transmission between the Moon and the Earth. Large amounts of observational data by many kinds of sensor and video data are transmitted during a mission, but commands and other information sent to the Moon are orders of magnitude less than that. Thus, only the data link from the Moon to the Earth is discussed in this section.

The KAGUYA lunar orbiter was launched by JAXA in 2007 in cooperation with the Japan Broadcasting Corporation and was equipped with a high-definition television (HDTV) camera. Its mission was to acquire detailed HDTV images of the entire Moon ${ }^{4}$. Beyond the scientific value of high-resolution images, high-quality lunar images have sparked considerable public interest in space exploration and science. As a successor of KAGUYA HDTV, JAXA Space Exploration Center (JSEC), an execution unit of the ARTEMIS/GATEWAY program in Japan, is requesting to equip 8K ultra HDTV camera on the lunar explorer.

Although only one HDTV camera was installed on the KAGUYA, several $8 \mathrm{~K}$ cameras will be installed on the lunar orbiter and at the lunar surface, including the Gateway, during the international manned lunar exploration. Moreover, it is expected that multiple $8 \mathrm{~K}$ videos will be transmitted to the Earth in real time, in contrast to the transmission of the KAGUYA's HDTV video which was delayed twenty times by store-and-forward transmitting over the $10 \mathrm{Mbps}$ X-band link ${ }^{4}$.

An 8K camera can acquire high-quality images, but the volume of data is enormous. The generated data rate of highdefinition imaging is at least $24 \mathrm{Gbps}$ and can reach a maximum of $144 \mathrm{Gbps}{ }^{5}$. The most often used transmission speed seems to be $48 \mathrm{Gbps}$. In this paper, the $8 \mathrm{~K}$ high-definition television data is assumed to be transmitted at $48 \mathrm{Gbps}$. Even if the $8 \mathrm{~K}$ video in the international joint manned lunar exploration is subject to delays on the order of the KAGUYA mission, this performance will be acceptable for raw data transmission of such tremendous data volume. In the terrestrial broadcasting (which has already begun), it is not easy to deliver such a large amount of 8K HDTV data to each household, thus it is transmitted after irreversible compression to 80-100 Mbps by H.265 in reference 6. Such lossy compressed image quality will be sufficient for monitoring and publicity on Earth, and real-time transmission will be essential.

From the above, the transmission requirements for an optical communication system for $8 \mathrm{~K}$ camera video from the lunar mission is as follows.

1. Real-time transmission of lossy compression $8 \mathrm{~K}$ video data

$100 \mathrm{Mbps} * 5-10 \mathrm{ch}=0.5-1 \mathrm{Gbps}$

2. Stored for delayed transmission of raw data for one channel of $8 \mathrm{~K}$ video data

2.4 Gbps (note: 1/20 store-and-forward transmission)

Under these assumptions, only $1 / 100$ th to $1 / 200$ th of the raw $8 \mathrm{~K}$ real image data for all 5 to 10 channels can be sent. Suppose the selection method is to check the lossy compressed video transmitted in real time in advance and choose the raw data to be sent. This approach would need many recording devices for each $8 \mathrm{~K}$ camera for at least several hours. Another method would decide in advance that only part of the raw data would be necessary. The acquisition, storage, and transmission of raw data would be limited to scientifically necessary data. 
In any case, it will be necessary to devise a method to make the recording device as small as possible by filming, storing, and transmitting partial data in a planned manner.

Furthermore, scientific data and housekeeping telemetry would be sent from the Gateway, lunar orbiter, lunar lander, and rover. Considering that $100 \mathrm{Base}-\mathrm{T}$ is the currently used data bus for experimental equipment in the Japanese Experiment Module of the ISS ${ }^{7}$, at least 1 Gbps Ethernet will be used in the Gateway Based on Gateway's official documentation ${ }^{8}$, Giga-Ethernet will be used as the data bus on Gateway. Therefore, we must assume that there will be requests to send data at up to $1 \mathrm{Gbps}$ in addition to $8 \mathrm{~K}$ video data.

These requirements will generate a total of 3.9 to 4.4 Gbps of user data during lunar missions. If the demand for raw data is low, it can be sent within the Ethernet's 1 Gbps. In this case, 1.5 to 2.0 Gbps user data will be created in Lunar missions.

Therefore, a total of 3.9 to $4.4 \mathrm{Gbps}$ (or 1.5 to $2 \mathrm{Gbps}$ ) of data needs to be sent from the Moon. Furthermore, since error correction codes and so on are added to this data volume, a modulation/demodulation speed (hereafter channel rate) of the link from the Moon must be about $5 \mathrm{Gbps}$ (or 2.5Gbps). From the above, in this paper, a channel rate of $5 \mathrm{Gbps}$ and $2.5 \mathrm{Gbps}$ is assumed to be the transmission requirement to the Earth of the future (assuming the 2030s) lunar mission. Moreover, large data transmission capacity, seamless and real-time $8 \mathrm{~K}$ data transmission (hereafter "real-time transmission") will also be needed because of demands of human bi-directional communication accompany with $8 \mathrm{~K}$ video data.

\section{PRELIMINARY STUDY OF LUNAR-EARTH OPTICAL COMMUNICATIONS LINK}

In this section, the author discusses the preliminary study results of the Lunar-Earth optical communications link.

The LADEE mission applied Pulse Position Modulation (PPM) method for communication. The PPM method applies a photon counter as a receiver in a typical Superconducting Single-Photon Detector (SSPD) and realizes a less than 1 photon per bit ultra-high sensitivity receiving and demodulating. The PPM method already been demonstrated by the LADEE lunar explorer using $622 \mathrm{Mbps}$ data transmission with SSPDs as OGS receivers. ${ }^{2}$ The $1550 \mathrm{~nm}$ band was the standard downlink from spacecraft in the Consultative Committee of Space Data System (CCSDS). ${ }^{9,10}$ However, realizing a faster channel rate than LADEE is challenging because the SSPD performance is limited. ${ }^{11}$

In the previous section, the author discussed using a channel rate of 2.5 or 5 Gbps for Moon-to-Earth data transmission. Since applying PPM at $2.5 \mathrm{Gbps}$ is impossible, the author suggests that the Phase Shift Keying (PSK) method is suitable for high-speed optical links from the Moon.

A PSK optical communication system uses the $1550 \mathrm{~nm}$ band, commonly employed in ground optical communications systems. Moreover, PSK optical communication system also applied in space optical communication systems as follows. On 29th November 2020, JAXA has launched the GEO optical data relay node called the Laser Utilized Communication System (LUCAS, [originally the Japan optical Data Relay System, JDRS] ${ }^{12}$ ). LUCAS uses a 1.8 Gbps optical link from low earth orbit (LEO) earth observation satellites to a GEO relay node with a Ka-band feeder link from GEO to the ground. NASA will launch the Laser Communication Relay Demo (LCRD) ${ }^{13}$ in 2021, which uses 1.224 Gbps data transmission (the channel rate is $2.88 \mathrm{Gbps}$ ). The European Data Relay System (EDRS) ${ }^{14}$ of ESA, under operation on orbit, equips a Laser Communication Terminal (LCE) using a $1064 \mathrm{~nm}$ Homodyne system for 1.8 Gbps user data transmission (a $2.8125 \mathrm{Gbps}$ channel rate), with a much higher data rate in the planning. ${ }^{15,16}$ PSK will meet the requirements for transmitting a 2.5 or 5 Gbps channel rate from the Moon.

Next to methods of optical communication, the author discusses the configurations of Lunar-Earth optical links. LADEE uses optical ground stations (OGSs) as Earth side communication terminals. The author calls this type of lunar optical 
communication system Type-1. The Type- 2 system will be used for spacecraft on and around the Moon to communicate with an optical data relay satellite on GEO. Figure 1 indicates Type-1 and -2 concept.

JSEC is planning to send $8 \mathrm{~K}$ video data from the Moon and also requests a planned Type- 2 system since the Type- 2 will have a higher certainty of real-time transmission. This section discusses the preliminary study results of Type-1 and Type-2 optical communications applying PSK using the $1550 \mathrm{~nm}$ band. The PSK method using the $1550 \mathrm{~nm}$ band is under an enactment in CCSDS in the Experimental Book (Orange Book) and is introduced in references. ${ }^{17,18}$

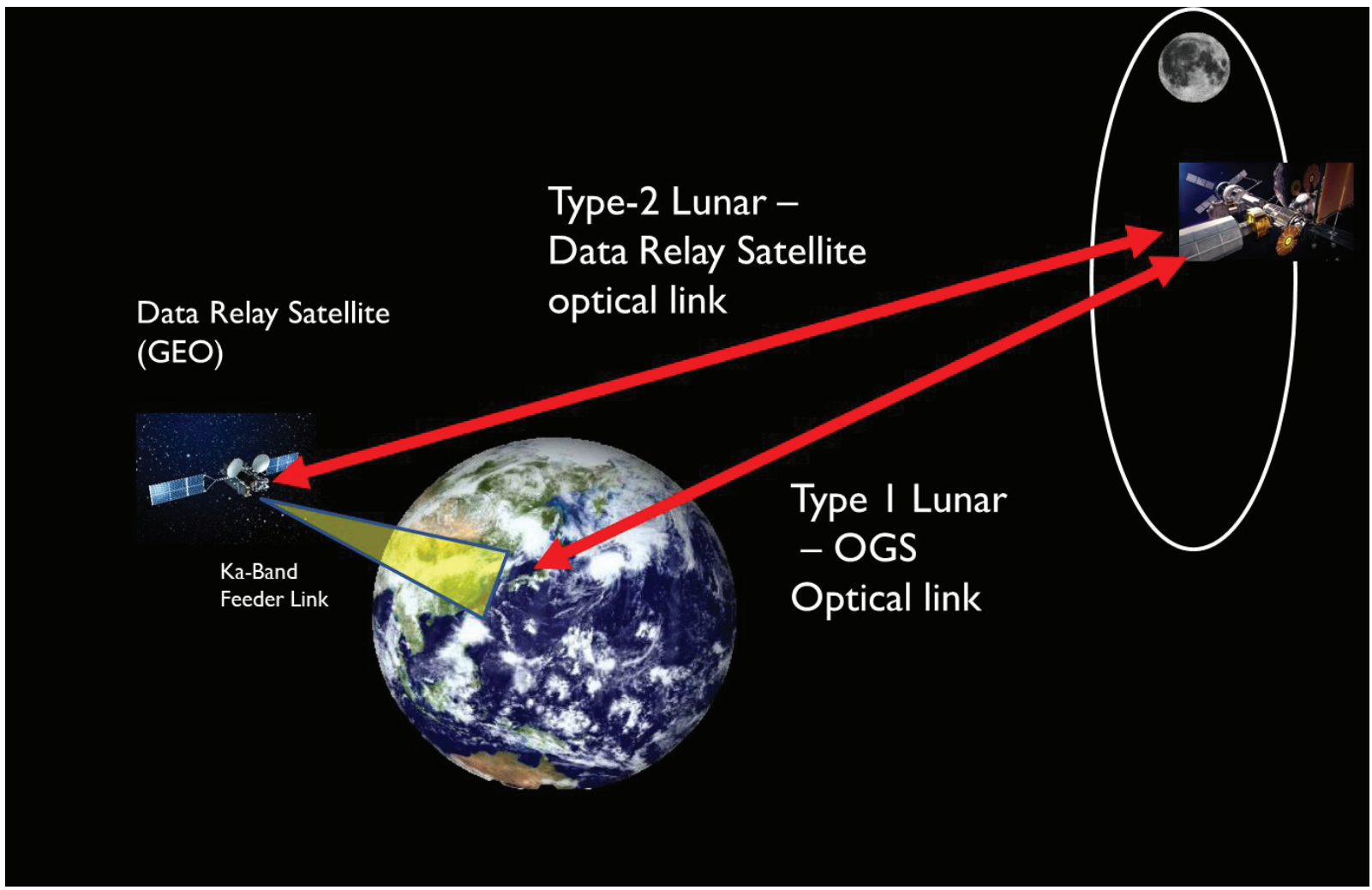

Figure 1 A Concept of 2 types of optical link from the Moon to the Earth

\subsection{Type-1: Lunar-OGSs optical link}

This subsection discusses the Type-1 lunar optical communications system. The PSK method is chosen as the communication method. Table 1 shows the tentative optical link budget between the Moon and OGS. The downlink will handle a huge amount of $8 \mathrm{~K}$ UHDTV data, but the uplink requirement is much smaller. The author assumes that the downlink channel rate is $2.5 \mathrm{Gbps}$ and that of the uplink channel is $39.1 \mathrm{Mbps}(2500 \mathrm{Mbps} / 64)$. To reject sunlight reflected from the Earth (during OGS daytime operation) and the Moon (during the lunar optical terminal fly over the Moon itself), an initial(coarse) acquisition sensor (CAS, i.e. InGaAs-QD) and a fine-tracking sensor (FTS, i.e. InGaAsQD) are equipped for a very narrow band of $0.5 \mathrm{~nm}$ optical bandpass filter (BPF) in this table. 
Table 1. Optical link budget between the Moon and OGS (tentative)

Use only communication beam (Down 1560nm, Up 1540nm)

\begin{tabular}{|c|c|c|c|c|c|c|}
\hline & \multicolumn{3}{|c|}{$\begin{array}{c}\text { Downlink(1560nm) } \\
\text { From Lunar to OGS }\end{array}$} & \multicolumn{3}{|c|}{$\begin{array}{c}\text { Uplink(1540nm) } \\
\text { From OGS to Lunar }\end{array}$} \\
\hline & $\begin{array}{l}\text { Initial } \\
\text { Acquisition }\end{array}$ & $\begin{array}{l}\text { Fine } \\
\text { Tracking }\end{array}$ & $\mathrm{COM}$ & $\begin{array}{l}\text { Initial } \\
\text { Acquisition }\end{array}$ & $\begin{array}{l}\text { Fine } \\
\text { Tracking }\end{array}$ & $\mathrm{COM}$ \\
\hline Wavelength(nm) & 1560 & 1560 & 1560 & 1540 & 1540 & 1540 \\
\hline Bit rate $(\mathrm{M}$ bps $)$ & - & - & 2500 & - & - & 39.1 \\
\hline Transmitter power $(\mathrm{dBm})$ & 40.0 & 40.0 & 40.0 & 46.0 & 40.0 & 40.0 \\
\hline Aperture of transmitter (m) & 0.09 & 0.09 & 0.09 & 0.14 & 0.14 & 0.14 \\
\hline $\operatorname{EIRP}(\mathrm{dBm})$ & 145.9 & 145.9 & 145.9 & 146.2 & 146.2 & 146.2 \\
\hline Distance $(\mathrm{km})$ & 380,000 & 380,000 & 380,000 & 380,000 & 380,000 & 380,000 \\
\hline Space Loss (dB) & -309.7 & -309.7 & -309.7 & -309.8 & -309.8 & -309.8 \\
\hline Atmospheric loss (dBm) & 6.0 & 6.0 & 6.0 & 6.0 & 6.0 & 6.0 \\
\hline Aperture of receiver (m) & 1.0 & 1.0 & 1.0 & 0.09 & 0.09 & 0.09 \\
\hline Receiver optical loss (dB) & -8.0 & -8.0 & -7.0 & -3.5 & -8.0 & -7.0 \\
\hline Received power (dBm) & -61.5 & -57.3 & -55.0 & -70.5 & -77.9 & -73.8 \\
\hline $\begin{array}{ll}\text { Minimum } & \text { Required } \\
\text { received power }(\mathrm{dBm})\end{array}$ & -62.3 & -74.5 & -56.5 & -71.0 & -78.2 & -74.5 \\
\hline Margin $(\mathrm{dB})$ & 0.8 & 17.2 & 1.5 & 0.5 & 0.3 & 0.7 \\
\hline
\end{tabular}

Table 1 indicates the following technical requirements:

A) A high-sensitivity optical receiver, with high-speed (2.5 Gbps) and low-rate (39.1 Mbps) capability. Table 1 assumes a 7 photons/bit at a bit error rate (BER) of 1e-3 during demodulation. Those values are challenging even if high performance the coherent digital receiver ${ }^{19}$ would be applied. Especially, low-rate has not be demonstrated even in the laboratory. The coherent digital receiver should also have a high-performance forward error correction code (FEC) like DVB-S2 ${ }^{16,17}$. These technologies are not mature under space environment (TLR=3), so further investigation and development will be needed.

B) Site diversity of OGSs. It is often necessary to combine several ground stations to operate efficiently, observe clouds, and perform optical communications when a station is cloud-free. Link availability must be $99 \%$ or more with the combination of three or more OGSs (even though an RF communication system can do this with a single antenna). According to studies from Japanese researchers, considering site diversity from meteorological satellite data using three stations in Japan, the link availability was $75 \%$ even at the best location ${ }^{20}$. They are studying the number and placement of OGSs to realize at $99 \%$ or higher link availability.

C) Optical high-power amplifier (HPA) onboard spacecraft does not have a mature technology. A $10 \mathrm{~W}(+40 \mathrm{dBm})$ optical HPA is under development. ${ }^{21,22}$ 


\subsection{Type-2; Lunar - GEO Data Relay Satellite optical link}

The following subsection investigates the optical link between the Moon and a GEO Data Relay Satellite. Table 2 shows the tentative optical link budget for one. As in the earlier subsection, the PSK method is assumed for the return and forward links. Also, channel rate and BPF of CAS and FTS were assumed as same as in the earlier subsection.

Table 2. Optical link budget between the Moon and GEO relay (tentative)

Use only communication beam (Down 1560nm, Up 1540nm)

\begin{tabular}{|c|c|c|c|c|c|c|}
\hline & Fr & Return & SEO & & $\begin{array}{l}\text { Forward } \\
\text { m GEO t }\end{array}$ & nar \\
\hline & $\begin{array}{l}\text { Initial } \\
\text { Acquisition }\end{array}$ & $\begin{array}{l}\text { Fine } \\
\text { Tracking }\end{array}$ & $\mathrm{COM}$ & $\begin{array}{l}\text { Initial } \\
\text { Acquisition }\end{array}$ & $\begin{array}{l}\text { Fine } \\
\text { Tracking }\end{array}$ & $\mathrm{COM}$ \\
\hline Wavelength(nm) & 1560 & 1560 & 1560 & 1540 & 1540 & 1540 \\
\hline Bit rate $(\mathrm{M} \mathrm{bps})$ & - & - & 2500 & - & - & 39.1 \\
\hline Transmitter power $(\mathrm{dBm})$ & 40.0 & 40.0 & 40.0 & 40.0 & 40.0 & 40.0 \\
\hline Aperture of transmitter (m) & 0.09 & 0.09 & 0.09 & 0.14 & 0.14 & 0.14 \\
\hline $\operatorname{EIRP}(\mathrm{dBm})$ & 143.3 & 143.3 & 143.3 & 146.2 & 146.2 & 146.2 \\
\hline Distance $(\mathrm{km})$ & 380,000 & 380,000 & 380,000 & 380,000 & 380,000 & 380,000 \\
\hline Space Loss $(\mathrm{dBm})$ & -309.7 & -309.7 & -309.7 & -309.8 & -309.8 & 0309.8 \\
\hline Aperture of receiver $(\mathrm{m})$ & 0.35 & 0.35 & 0.35 & 0.09 & 0.09 & 0.09 \\
\hline Receiver optical loss (dB) & -10.0 & -5.0 & -5.0 & -3.5 & -8.0 & -7.0 \\
\hline Received power $(\mathrm{dBm})$ & -65.6 & -57.5 & -56.1 & -70.8 & -71.9 & -67.5 \\
\hline 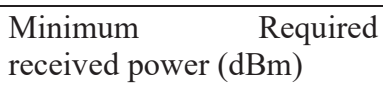 & -67.4 & -74.6 & -56.5 & -71.0 & -78.2 & -74.5 \\
\hline $\operatorname{Margin}(\mathrm{dB})$ & 1.8 & 17.1 & 0.4 & 0.2 & 6.3 & 7.0 \\
\hline
\end{tabular}

Table 2 presents some technical challenges as well:

A) A high-sensitivity optical receiver with high-speed (2.5 Gbps) and low-rate (39.1 Mbps) capabilities. The issues are the same as in subsection $3.1 \mathrm{~A}$ ); moreover, a $2.5 \mathrm{Gbps}$ demodulator will be on the GEO satellite. Realizing this link is much more complicated than $3.1 \mathrm{~A}$ ) is, where the receiver is ground-based. The return link needs to be coded for high performance. Soft-decision FEC (i.e., DVB-S2) will be decoded on the ground since it is difficult to decode Gbps-order, high-performance, soft-decision FEC onboard.

B) Large optical antenna for the GEO data relay with $35 \mathrm{~cm}$ diameter. $35 \mathrm{~cm}$ diameter or larger optical antenna is usual for passive optical sensor, since highly precise laser transmission direction control is included in space optical communication, an optical antenna must have a higher transmitted wave surface accuracy and alignment stability than a passive optical sensor. In most cases, an optical antenna on a 2-axis gimbal or mirror gimbal is driven by a motor, and an angle encoder is also needed for pointing for a counter optical terminal. Moreover, since this optical antenna is onboard equipment, it must be stiff and lightweight. Therefore, developing a $35 \mathrm{~cm}$ optical antenna for space optical communication is still challenging. The already developed optical antenna from KIRARI (OICETS) is $26 \mathrm{~cm}^{23}$, and that of LUCAS is only $14 \mathrm{~cm}^{12}$.

C) The $10 \mathrm{~W}(+40 \mathrm{dBm})$ onboard optical HPA is the same as in subsection $3.1 \mathrm{C})$. 


\section{RESULTS OF THE TRADE-OFF STUDY: OGS VS. GEO RELAY}

Up to the previous section, we have described the needs for data transmission from the Moon and the preliminary examination results of the two types of optical link called Type-1 and -2 when transmitting this by the PSK method, and described the technical issues for realization. In this section, we will discuss and compare the development and operation aspects of Type- 1 and -2 shown in the previous section.

To realize a stable Earth-Lunar optical link, at least ten OGSs worldwide will be required in case of Type-1 as discussed in this section, or in case of Type-2, GEO data relay, will require at least two GEO nodes. At development point of view, over USD 100 million will likely be required for the initial setup of a GEO relay. Some million USD will be used in constructing an OGS; an OGSs network system will be less expensive than using a GEO relay.

At operation aspect, considering the Earth's rotation, this combination of OGS network systems will be required:

- $\quad 3$ or more OGSs network systems in East Asia and Oceania continent,

- $\quad 3$ or more OGSs network systems in the Americas continent, and

- $\quad 3$ or more OGSs network systems in Europe and Africa continent.

Each network must be over $99 \%$ available for optical links to avoid cloud cover. OGSs network operation must be done with frequent and unexpected handovers between OGSs to avoid cloud coverage. Since each handover must accompany a Pointing, Acquisition, and Tracking (PAT) sequence that interrupts communication, the OGSs-Lunar link will be interrupted often. This means that optical links using OGS do not meet the future requirement of real-time data transmission. Therefore, despite the high cost, the Type-2, GEO relay, has two advantages over Type-1, OGSs network:

A GEO relay will operate under a planned schedule, including switching, since spacecraft orbit is precisely predicted. This also means that GEO relays already satisfy the requirement of real-time data transmission.

A GEO relay will never be disturbed by clouds.

Concerning the GEO relay's expense, the total cost is expected to drop as the lunar GEO relay system is developed together with a GEO optical data relay system for LEO satellites.

\section{SUMMARY AND CONCLUSION}

This paper discusses the required data transmission rate needed for a link from the Moon to the Earth. Results showed that a minimum $2.5 \mathrm{Gbps}$ channel rate would be needed to transmit multi-channel compressed 8K UHDTV video data and 1 Giga Ethernet in the Gateway. Also discussed are lunar-OGS optical and Lunar-GEO relay satellite optical communication types, with a preliminary budget. The technical issues to be solved to realize the project within budget are extracted and discussed.

Based on the above issues, the author presents his perspective on lunar optical communication here, including his expectations. At first, optical communication between the Moon and OGS will be realized in the next ten years. The optical communication system between the Moon and OGS involves frequent handovers between OGSs under network control, so frequent optical link outages will hinder real-time data transmissions, like 8K UHDTV live video.

In the decade of 2030, the demand for 8K UHDTV video data will increase until real-time data transmission will become obligatory for an Earth-lunar optical link. Therefore, a highly reliable and stable GEO relay-lunar optical link must be realized by that date.

\section{REFERENCES}

[1] https:/www.nasa.gov/feature/multilateral-coordination-board-joint-statement

[2] D. M. Boroson, B. S. Robinson, D. V. Murphy, D. A. Burianek, F. Khatri, "Overview and Results of the Lunar Laser Communication Demonstration”, Proc. of SPIE Vol. 89710S (2014) 
[3] https://www.nasa.gov/directorates/heo/scan/services/networks/deep_space_network/

[4] https://www.kaguya.jaxa.jp/index_e.htm

[5] ARIB STD-B58, https://www.arib.or.jp/english/std_tr/broadcasting/std-b58.html

[6] ISO/IEC 23008-2 / ITU-T H.265 "High Efficiency Video Coding"

[7] "KIBO handbook" https://iss.jaxa.jp/kibouser/library/item/kibo_handbook_en.pdf

[8] GP10003, "GATEWAY PROGRAM SUBSYSTEM SPECIFICATION FOR COMMAND AND DATA HANDLING (C\&DH)", NASA document

[9] Recommended STANDARS 141.0-B-1, "OPTICAL COMMUNICATIONS PHYSICAL LAYER", https://public.ccsds.org/Publications/BlueBooks.aspx

[10] Recommended STANDARS 142.0-B-1, "OPTICAL COMMUNICATIONS CODING AND SYNCRONYZATION", https://public.ccsds.org/Publications/BlueBooks.aspx

[11] Robinson, B. S., Kerman, A. J., Dauler, E. A., Barron, R. J., Caplan, D. O., Stevens, M. L., Carney, J. J. and Hamilton, S. A., "781Mbpt/s Photon-counting optical communications using a superconducting nanowire detector" OPTICS LETTERS, vol. 31-4, pp444-446 (2006)

[12] Chishiki, Y. Yamakawa, S., Takano, Y., Miyamoto, Y., Araki, T., Kohata, H., "Overview of optical data relay system in JAXA", Proc. of SPIE Vol. 97390D, (2016)

[13] Edwards, B. L., Israel, D. J., Vithlani, S. K., "Latest Changes to NASA's Laser Communications Relay Demonstration Project", Proc. SIPE Vol. 105240P (2018)

[14] Heine, F., Sanchez-Tercero, A., Martin-Pimentel, P., Höpcke, N., Hasler, D., Marynowski, T., Zech, H., Alber, L., Klement, J., "Status of Tesat laser communication activities", Proc. of SPIE Vol. 1127204, (2020)

[15] Araki, T., "A Study of the Future Optical Data Relay System; Requirements, Problems and Solution" Proceedings of 2017 IEEE International Conference on Space Optical Systems and Applications (ICSOS)

[16] Hauschildt, H., Elia, C., Moeller H. L., Armengol, J. M. P. "HydRON: High thRoughput Optical Network", Proc. of SPIE Vol. 109100K (2019)

[17] Edwards, B. L., Schulz K.-J., Hamkins, J., Robinson, B., Alliss, R., Daddato, R., Schmidt, C.,Gigenbach, D., and Braatz, L., "An Update on the CCSDS Optical Communications Working Group" Proc. of IEEE International Conference on Space Optical Systems and Applications (ICSOS) pp1-9, 2017 (2017).

[18] Edwards, B. L., Schulz, K.-J., Hamkins, J., Robinson, B., Alliss, R., Daddato, R., Gigenbach, D., and Braatz. L., "An Update on the CCSDS Optical Communications Working Group and Interoperability Standards" Proc. of IEEE International Conference on Space Optical Systems and Applications (ICSOS) pp1-6, 2019 (2019)

[19] Araki, T., "Digital Coherent Receiver Technique for Onboard Receiver of Future Optical Data Relay System" Proc. of SPIE Vol. 973919 (2016)

[20] Ueda, Y., Mukai, T., Takayama, Y., "Studies on site diversity to mitigate cloud blockage in satellite-ground optical communications by long term ground meteorological observation data", 37th International Communications Satellite Systems Conference (ICSSC), ICSSC-05, \#147, pp.1-7

[21] Haddad, E., Gonthier, F., Peng, Q., Poenariu, V., Tagziria, K., Lavoie, J., Murzionak, P., Schinn, G., Karafolas, N., Bringer, C., "10W single-mode PM optical amplifiers in the 1.5- $\mu \mathrm{m}$ region for space applications" Proc. of SPIE Vol. 1118035 (2018)

[22] Haddad, E., Limodehi, H., Saffarpour, A., Peng, Q., Tagziria, K., Murzionak, P., Schinn, G., Karafolas, N., "Space Qualification of a 10W single-mode PM optical amplifiers in the $1.5-\mu \mathrm{m}$ regio", ICSO2020 233, to be published.

[23] Jono, T., Takayama, Y., Shiratama, K., Mase, I., Demelenne, B., Sodnik, Z., Bird, A., Toyoshima, M., Kunimori, H., Giggenbach, D., Perlot, N., Knapek, M., and Arai, K., "Overview of the inter-orbit and orbit-toground laser communication demonstration by OICETS" Proc. of SPIE Vol. 645702, (2007) 